\title{
Abiraterone acetate: oral androgen biosynthesis inhibitor for treatment of castration-resistant prostate cancer
}

This article was published in the following Dove Press journal:

Drug Design, Development and Therapy

I3 January 201 I

Number of times this article has been viewed

\author{
Yasser Rehman' \\ Jonathan E Rosenberg \\ 'Division of Hospital Medicine, UMass \\ Memorial Healthcare, Worcester, MA, \\ USA; ${ }^{2}$ Lank Center for Genitourinary \\ Oncology, Dana Farber Cancer \\ Institute, Harvard Medical School, \\ Boston, MA, USA
}

\begin{abstract}
Prostate cancer is the second leading cause of cancer death in men in the US and Europe. The treatment of advanced-stage prostate cancer has been androgen deprivation. Medical castration leads to decreased production of testosterone and dihydrotestosterone by the testes, but adrenal glands and even prostate cancer tissue continue to produce androgens, which eventually leads to continued prostate cancer growth despite castrate level of androgens. This stage is known as castrate-resistant prostate cancer (CRPC), which continues to be a challenge to treat. Addition of androgen antagonists to hormonal deprivation has been successful in lowering the prostate-specific antigen levels further, but has not actually translated into life-prolonging options. The results of several contemporary studies have continued to demonstrate activation of the androgen receptor as being the key factor in the continued growth of prostate cancer. Blockade of androgen production by nongonadal sources has led to clinical benefit in this setting. One such agent is abiraterone acetate, which significantly reduces androgen production by blocking the enzyme, cytochrome P450 17 alpha-hydroxylase (CYP17). This has provided physicians with another treatment option for patients with CRPC. The landscape for prostate cancer treatment has changed with the approval of cabazitaxel, sipuleucel-T and abiraterone. Here we provide an overview of abiraterone acetate, its mechanism of action, and its potential place for therapy in CRPC.
\end{abstract}

Keywords: CRPC, abiraterone, CYP17, inhibitors, androgens, castration resistant prostate cancer

\section{Introduction}

Prostate cancer is widely known to be dependent on androgens. The testes secrete testosterone which accounts for $95 \%$ of androgens in the body while the remainder is produced in the adrenal glands. ${ }^{1,2}$ The majority of testosterone is primarily bound to albumin and sex hormone binding globulin, though some is transported as free testosterone in serum. Upon entering the testes, free testosterone is converted to dihydrotestosterone by the enzyme $5 \alpha$-reductase. Dihydrotestosterone has a five-fold affinity for the androgen receptor compared with testosterone. Prostate cancer growth is dependent on levels of androgen in the body which help maintain the balance in favor of cell growth over apoptosis. ${ }^{3}$ Recently, the treatment of CRPC has changed dramatically with the approval of sipuleucel-T, cabazitaxel, denosumab, and abiraterone acetate by the US Food and Drug Administration.

Cabazitaxel is a semisynthetic taxane analog designed to overcome taxane resistance. Preclinical studies demonstrated cabazitaxel to overcome docetaxel-resistant cell lines. Phase I studies further demonstrated cabazitaxel to show efficacy not only
Correspondence: Yasser Rehman UMass Memorial Healthcare, Division of Hospital Medicine, II 9 Belmont Street, Worcester MA 01605, USA $\mathrm{Tel}+\mathrm{I} 6464022900$

Email yasser_rehman@dfci.harvard.edu 
in chemotherapy-naïve CRPC but also docetaxel-pretreated CRPC. Based on the encouraging results, a Phase III trial was designed whereby 755 docetaxel pretreated patients were randomized to cabazitaxel plus prednisone every three weeks versus mitoxantrone plus prednisone arms every three weeks for 10 cycles. Patients in the cabazitaxel arm demonstrated a survival benefit of 15.1 months versus 12.7 months in the cabazitaxel arm. The Food and Drug Administration has approved cabazitaxel for the treatment of prostate cancer. ${ }^{4}$

Sipuleucel-T is an autologous immunotherapy which was approved by the Food and Drug Administration recently for treatment of metastatic CRPC. Sipuleucel-T is prepared by removing mononuclear cells, including antigen-presenting cells by therapeutic leukopheresis and incubating them with a combination of cytokines (granulocyte-macrophage colony-stimulating factor) and prostatic acid phosphatase. Patients undergo leukapheresis at 0,2, and, 4 weeks with reintroduction of the vaccine 2 days later. A Phase III trial of sipuleucel-T versus placebo in symptomatic or mildly symptomatic metastatic CRPC demonstrated an overall survival benefit of 4.1 months in the sipuleucel-T arm and a 3-year overall survival of $31.7 \%$ in the sipuleucel-T arm versus a $23 \%$ survival in the placebo arm, though rates of objective and prostate-specific antigen responses were very low. ${ }^{5}$

\section{Androgen receptor and signaling}

Androgen signaling is mediated through the androgen receptor, a ligand-dependent transcription factor located on the $\mathrm{X}$ chromosome. The receptor is composed of an amino terminal activating domain, a carboxy-terminal ligand binding domain, a hinge region, and a DNA binding domain. The inactive form of the androgen receptor resides within the cytoplasm and remains bound to heat shock proteins that prevent androgen receptor activation. The binding of androgens to the receptor leads to dissociation of the heat shock proteins and receptor phosphorylation which, in turn, leads to nuclear translocation, allowing for transcription of androgen-dependent genes. ${ }^{6}$

A number of different mechanisms appear responsible for castration resistance in prostate cancer. One of them is an androgen receptor mutation which is seen occasionally in advanced disease. ${ }^{7}$ Some androgen receptor mutations in CRPC make the androgen receptor lose its specificity to androgens and it becomes more responsive to estrogens, progesterone, and even antiandrogens. ${ }^{8}$ This can lead to prostate cancer growth despite castrate levels of testosterone. Occasionally, prostate cancer with resistance to flutamide will still respond to other antiandrogens like bicalutamide, suggesting that mutations in the androgen receptor are a direct result of selective pressure. ${ }^{9}$ Androgen receptor hyperresponsiveness can also result from androgen receptor gene amplification. Copy number gains of the androgen receptor allow continued prostate cancer growth despite low androgen levels. Androgen receptor gene amplifications are noted in $28 \%$ of recurrent therapy-resistant prostate cancer patients exposed to androgen ablation, but has not been observed in early tumor samples without exposure to androgen ablation. ${ }^{10}$ This suggests the possibility of clonal selection of cancer cells with the ability to grow despite very low levels of circulating androgens. Upregulation of nuclear transcription coactivators also allows androgen receptor-mediated gene transcription despite low levels of circulating androgens or presence of less potent androgens. ${ }^{11}$ In addition, prostate cancer cells have been shown to upregulate intratumoral steroidogenesis to maintain intratumor androgen at levels required for tumor growth. ${ }^{12}$ This mechanism leads to tumor growth despite castrate level androgens in the body. In addition, the fusion of androgen-activated TMPRSS2 with the ETS family of oncogenes ERG or ETV1 has been noted to occur frequently in prostate cancer. The precise role of TMPRSS2/ETS translocations in castration resistance is unclear at this time, although androgen-regulated ERG transcription is reactivated in the setting of androgen receptor reactivation in castration resistance, and may contribute to progression of CRPC. ${ }^{13}$

\section{Cytochrome PI 7 enzyme}

The CYP17 enzyme is a cytochrome P450 (CYP) enzyme. Located in the endoplasmic reticulum of the testis, ovaries, adrenals, and placenta, CYP17 drives the synthesis of glucocorticoids and sex hormones. The enzyme has both $17 \alpha$ hydroxylase and $\mathrm{C}_{17,20}$ lyase activity, and plays a critical role in the production of cortisol and androgen synthesis (Figure 1). Cytochrome b5 regulates the activity of $\mathrm{CYP}_{17,20}$ lyase. A high b5/CYP ${ }_{17,20}$ lyase ratio usually occurs in the testes, which leads to production of androgens, while a low b5/c lyase ratio in adrenal glands will ensure cortisol production. ${ }^{14,15}$

\section{Role in androgen production}

Production of androgens or cortisol in the body begins by conversion of cholesterol into pregnenolone which can then be further converted to androgens or progesterone via $3 \beta$ hydroxysteroid dehydrogenase. $17 \alpha$ hydroxylase then converts progesterone into $17 \alpha$ hydroxyprogesterone and pregnenolone into $17 \alpha$ hydroxypregnenolone. $\mathrm{C}_{17,20}$ lyase then converts $17 \alpha$ hydroxyprogesterone and $17 \alpha$ hydroxypregnenolone into androstenedione and dehydroepiandrosterone, respectively. Androstenedione 


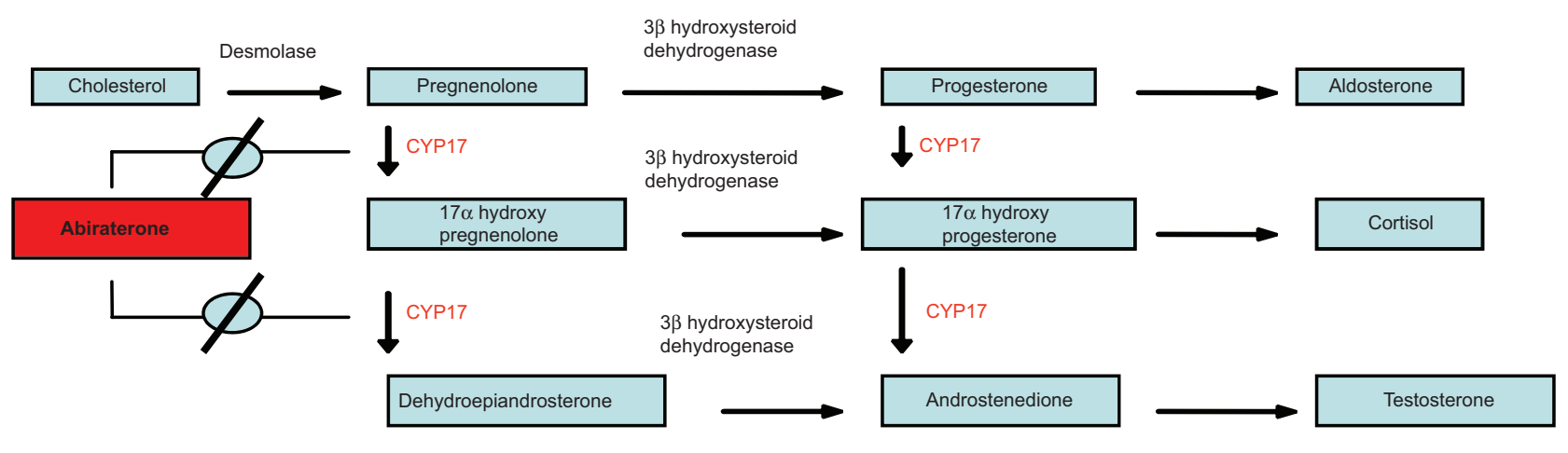

Figure I Schematic diagram of steroid synthesis.

Note: Role of CYPI7 at key enzymatic steps and its inhibition by abiraterone acetate.

and dehydroepiandrosterone are eventually converted into testosterone by enzymatic pathways. The enzyme $5 \alpha$ reductase then converts testosterone into dihydrotestosterone. Abiraterone inhibits $\mathrm{C}_{17,20}$ lyase irreversibly.

\section{Role in cortisol production}

$17 \alpha$ hydroxypregnenolone and $17 \alpha$ hydroxyprogesterone are also converted to cortisol precursors. Adrenocorticotropic hormone (ACTH) acts as a stimulus for converting cholesterol into pregnenolone, and is kept in check via a negative feedback loop by an increased cortisol level. By blocking the activity of $17 \alpha$ hydroxylase, cortisol production is diminished and the negative feedback effect on ACTH is removed. This leads to increased production of ACTH which, in turn, stimulates production of precursors above the $17 \alpha$ hydroxylase level. One of these precursors is corticosterone which is only a weak inhibitor of ACTH; high levels of corticosterone are required to inhibit the production of ACTH. The increased production of corticosterone leads to fluid retention, hypokalemia, and hypertension. These effects can be prevented by concomitant administration of low doses of prednisone, corticosterone, or dexamethasone.

\section{CYPI 7 inhibition in prostate cancer}

Inhibition of adrenal androgen steroid synthesis via CYP17 in men with prostate cancer has led to clinical antitumor responses. Use of ketoconazole and aminoglutethamide has been associated with prostate-specific antigen declines and measurable disease responses in some CRPC patients; these agents also have significant toxicity and are not associated with a survival benefit. ${ }^{16}$ However, these data suggested that a better inhibitor with less toxicity and more durable efficacy might lead to significant clinical benefit in CRPC. Abiraterone acetate was identified to be a potent and selective irreversible inhibitor of CYP17. Preclinical studies with abiraterone demonstrated reduction in androgen production downstream of CYP17 which resulted in decreased weight of the ventral prostate, testis, and seminal vesicles in mice. ${ }^{17}$ The first-in-human studies demonstrated a dose of $800 \mathrm{mg}$ once daily to be effective in suppressing testosterone to below detectable levels in humans and also provided a preliminary insight into its safety profile. ${ }^{18}$

\section{Phase I studies}

Based on the clinical data suggesting the importance of CYP17 in androgen synthesis and the activity of abiraterone in preclinical models, Phase I studies of this agent were initiated. One study evaluated 21 CRPC patients who received escalating doses of abiraterone from $250 \mathrm{mg}$ to $2000 \mathrm{mg}$. Antitumor activity was noted at all levels. However, due to a plateau effect in pharmacokinetics noted at $1000 \mathrm{mg}$, this dose was selected for further drug development. Decreases in testosterone, estradiol, and androgenic steroids downstream of CYP17 blockade were noted in all patients, as well as an increase in ACTH and steroids upstream of the blockade. Declines in prostate-specific antigen of $\geq 30 \%, \geq 50 \%$, and $\geq 90 \%$ were observed in 14 (66\%), 12 (57\%), and six $(29 \%)$ patients, respectively. A reduction in pain medication requirement and radiologic regression were noted in many patients. Adverse effects, mainly fluid retention, hypokalemia, and lower extremity edema, were easily reversed with eplerenone (a mineralocorticoid receptor antagonist). Interestingly, six patients in the study had an ERG rearrangement. Five of these patients responded well to treatment with abiraterone. ERG rearrangements can potentially be used in the future as a predictor of favorable outcome in response to abiraterone. ${ }^{19}$ This study also looked at the use of dexamethasone to test the hypothesis that potential resistance to abiraterone could be overcome with steroids by inhibiting ACTH and also to prevent upstream steroid production from stimulating a 


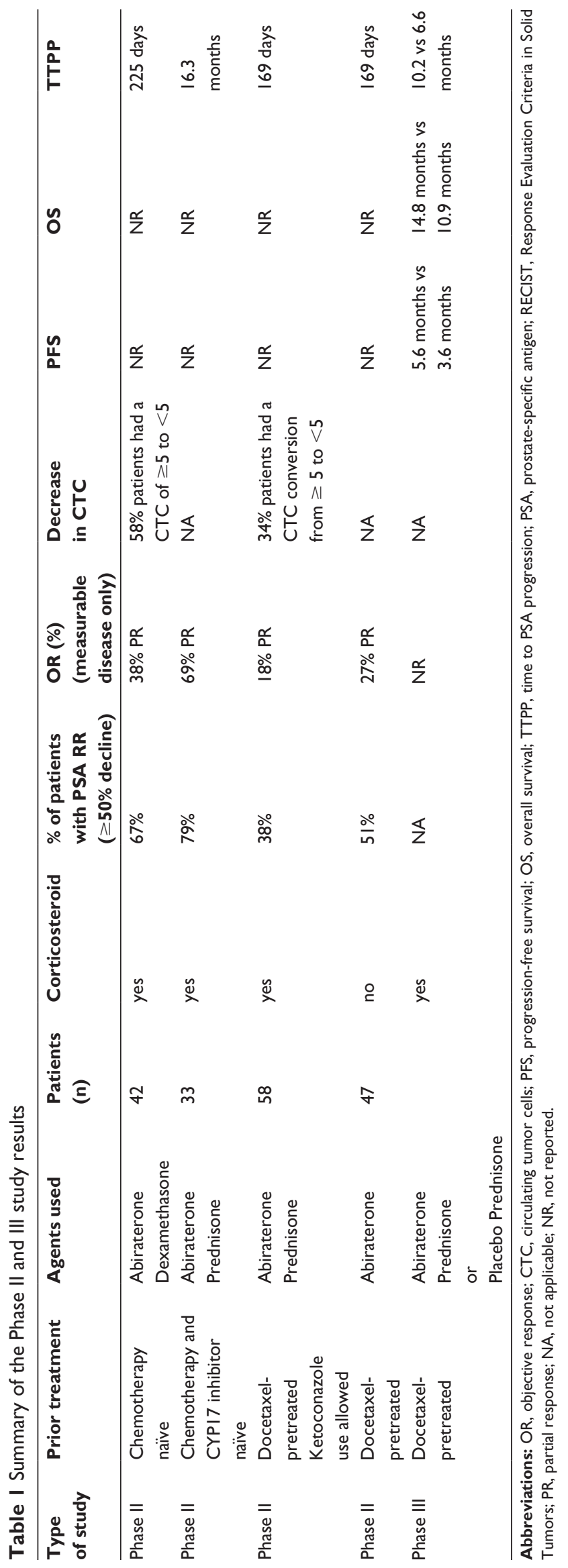

promiscuous androgen receptor. During the study, four of $15(26 \%)$ patients did respond to the addition of dexamethasone and became resensitized to abiraterone. ${ }^{19}$

Another Phase I study of 33 chemotherapy-naïve CRPC patients, some of who had previously been treated with ketoconazole, evaluated the response to escalating doses of abiraterone acetate. Eighteen patients (55\%) demonstrated a prostate-specific antigen decline of $\geq 50 \%$. In addition, this study demonstrated that abiraterone could overcome ketoconazole resistance, since $47 \%$ of ketoconazole-pretreated patients experienced $\geq 50 \%$ prostate-specific antigen declines, and confirmed the $1000 \mathrm{mg}$ daily dose of abiraterone acetate for future studies. ${ }^{20}$

\section{Phase II studies}

Two Phase II studies evaluated the anticancer activity and safety of abiraterone. One study enrolled 42 chemotherapynaïve CRPC patients with a primary endpoint of a $\geq 50 \%$ decline in prostate-specific antigen. $\mathrm{A} \geq 50 \%$ decline in prostate-specific antigen was observed in $28(67 \%)$ patients and a $\geq 90 \%$ decline of prostate-specific antigen was observed in eight (19\%) patients. Addition of dexamethasone to abiraterone at disease progression was noted to lead to confirmed secondary $\geq 50 \%$ prostate-specific antigen declines in $33 \%$ of patients. Twenty-four patients had measurable disease according to the Response Evaluation Criteria in Solid Tumors, and nine patients (37.5\%) showed tumor regression on computed tomography scans. ${ }^{21}$

Until recently, patients previously treated with docetaxel had few proven treatment options. A Phase II trial tested the efficacy of abiraterone monotherapy in 47 CRPC patients previously treated with docetaxel. Prostate-specific antigen declines of $\geq 30 \%, \geq 50 \%$, and $\geq 90 \%$ were seen in $68 \%$, $51 \%$, and $15 \%$ of patients, respectively. This study demonstrated that abiraterone has potent antitumor activity in patients treated with docetaxel. ${ }^{22}$ The common side effects noted in this study were hypokalemia, hypertension, and fluid retention, which were able to be managed with eplerenone.

To overcome these side effects of secondary hyperaldosteronism associated with single-agent abiraterone, low-dose prednisone was added to abiraterone in another study of docetaxel-pretreated CRPC. Fifty-eight men were enrolled, of whom 27 had prior exposure to ketoconazole. Twenty-two patients (36\%) experienced a $\geq 50 \%$ decline in prostate-specific antigen which also included 14 (45\%) of 31 ketoconazole-naïve and seven (26\%) of 27 ketoconazole-pretreated patients. ${ }^{23}$ While similar side effects were noted in this study compared with abiraterone 
studies without prednisone, the incidence of these side effects was significantly reduced.

Another Phase II study assessed the efficacy of abiraterone in CRPC patients naïve to both chemotherapy and ketoconazole. Along with prostate-specific antigen, serial changes in bone scan lesions were used to assess the disease process. The study not only demonstrated the efficacy of abiraterone in CRPC, but also highlighted the discordance of bone scan intensity and disease progression. The study was designed to include 33 CRPC patients who received $1000 \mathrm{mg}$ of abiraterone daily along with $5 \mathrm{mg}$ of prednisone twice daily in 28-day cycles. Bone scan "flare" was defined as disease progression by a radiologist after 3 months of treatment in the context of a $\geq 50 \%$ decline in prostate-specific antigen, with scan improvement or stability after an additional 3 months. Median time on therapy was 63 weeks and time to prostatespecific antigen progression was 16.3 months. Twenty-two of the 33 patients $(67 \%)$ showed a decline in prostate-specific antigen of $\geq 50 \%$ within 12 months. Overall, 26 of 33 patients (79\%) showed a $\geq 50 \%$ decline in prostate-specific antigen. Twelve of 23 (52\%) patients demonstrated a bone scan flare, of which 11 showed eventual improvement in bone scan over time. The side effects noted in the study were mainly grade $1 .{ }^{24}$ The results of the above mentioned studies are summarized in Table 1 along with the Phase III study results.

Despite significant antitumor activity demonstrated by abiraterone, as evidenced by a declining prostate-specific antigen level, it was noted that the decline was short-lived, and the prostate-specific antigen ultimately increased. This could potentially be explained by the fact that although abiraterone blocks androgen synthesis at the CYP17 level, androgens upstream continue to accumulate and can potentially stimulate an altered androgen receptor. This was demonstrated by Zhao et al in their study of prostate cancer cells in a patient resistant to androgen ablation therapy. The androgen receptor contained a double mutation which had high affinity for cortisol/cortisone, leading to increased prostate-specific antigen production. These sorts of alterations lead to the possibility that increased hormone levels upstream of CYP17 blockade may cause prostatespecific antigen rises after an initial decline. However, more work needs to be done to explore this theory further. ${ }^{25}$

\section{Phase III studies}

Based on the encouraging results of the above studies, a Phase III trial was initiated in April 2008 which randomized 1195 patients with docetaxel-refractory CRPC to either abiraterone or placebo in a 2:1 fashion; both arms received prednisone $5 \mathrm{mg}$ twice daily. Based on the recommendations of an independent data monitoring committee, the study was unblinded on August 20, 2010. At this point, the abirateronetreated patients had received a median of eight 28-day cycles, and the patients in the placebo arm had received a median four 28-day cycles. The study demonstrated a median overall survival in the abiraterone-treated arm of 14.8 months compared with 10.9 months in the placebo arm (hazards ratio 0.646, 95\% confidence interval: $0.54-0.77 ; P<0.0001$ ). The abiraterone arm also yielded superior outcomes in time to prostate-specific antigen progression (10.2 months versus 6.6 months, $P<0.0001)$, radiographic progression-free survival (5.6 months versus 3.6 months $P<0.0001$ ), and prostate-specific antigen declines $\geq 50 \%$ (confirmed, $29.1 \%$ versus $5.5 \%, P<0.0001) .{ }^{26}$ Based on these data, abiraterone acetate was approved by the Food and Drug Administration on April 28, 2011. The recommended dose is $1000 \mathrm{mg}$ daily along with prednisone $5 \mathrm{mg}$ twice daily. The most common adverse effects seen on abiraterone therapy were joint discomfort, hypertension, and hypokalemia.

\section{Ongoing studies}

Currently, a Phase II study is looking at the addition of dutasteride to abiraterone in metastatic CRPC. This study will be looking at mechanisms of androgen receptor resistance to abiraterone, as well as the effects of a combination of abiraterone/dutasteride on levels of testosterone, dihydrotestosterone at baseline, and progression. It will also look at the effect of the combination on the toxicity profile and duration of prostate-specific antigen response. Another trial is underway looking at the addition of sunitinib or dasatinib to abiraterone acetate for the treatment of prostate cancer. Additional Phase III studies are investigating abiraterone in other contexts. One trial is currently looking at the comparison of abiraterone plus prednisone versus placebo and prednisone in asymptomatic or mildly symptomatic metastatic CRPC who have not received chemotherapy.

\section{Conclusion}

The recent Food and Drug Administration approvals of cabazitaxel, sipuleucel-T, and abiraterone acetate for the treatment of CRPC have provided clinicians with much needed additional treatment options for prostate cancer. The exact sequencing of these agents in CRPC treatment requires further evaluation. Both cabazitaxel and abiraterone have shown survival benefits in docetaxel-pretreated patients. The appropriate sequencing of abiraterone and cabazitaxel is not known at this time. Given the toxicity profiles of both agents, patients with significant docetaxel-associated toxicity might benefit from a break from 
cytotoxic chemotherapy and be guided towards abiraterone. Other patients with disease progression but excellent performance status and more modest chemotherapy-associated toxicity might be best served by further highly active chemotherapy. The recent approval of abiraterone and its proven efficacy in docetaxel-pretreated patients provides yet another treatment option for this patient population. Ongoing trials will evaluate whether abiraterone leads to a survival benefit in patients with chemotherapy-naïve CRPC. The activity of abiraterone in prostate cancer suggests that it should also be explored as part of adjunctive hormonal therapy in localized prostate cancer to improve cure rates in high-risk patients. Localized prostate cancer trials are just beginning to be launched and will take many years to demonstrate benefits.

\section{Disclosure}

Dr Rosenberg has served as a consultant for Johnson and Johnson, Inc. Dr Rehman has no conflicts of interest to declare.

\section{References}

1. Denis LJ, Griffiths K. Endocrine treatment in prostate cancer. Semin Surg Oncol. 2000;18(1):52-74.

2. Mohler JL, Gregory CW, Ford OH III, et al. The androgen axis in recurrent prostate cancer. Clin Cancer Res. 2004;10(2):440-448.

3. Colombel M, Olsson CA, Ng PY, Buttyan R. Hormone-regulated apoptosis results from reentry of differentiated prostate cells onto a defective cell cycle. Cancer Res. 1992;52(16):4313-4319.

4. de Bono JS, Oudard S, Ozguroglu M, et al. Prednisone plus cabazitaxel or mitoxantrone for metastatic castration-resistant prostate cancer progressing after docetaxel treatment: a randomised open-label trial. Lancet. 2010;376(9747):1147-1154.

5. Higano CS, Schellhammer PF, Small EJ, et al. Integrated data from 2 randomized, double-blind, placebo-controlled, phase 3 trials of active cellular immunotherapy with sipuleucel-T in advanced prostate cancer. Cancer. 2009;115(16):3670-3679.

6. Balk SP. Androgen receptor as a target in androgen-independent prostate cancer. Urology. 2002;60(3 Suppl 1):132-138.

7. Marcelli M, Ittmann M, Mariani S, et al. Androgen receptor mutations in prostate cancer. Cancer Res. 2000;60(4):944-949.

8. Taplin ME, Bubley GJ, Shuster TD, et al. Mutation of the androgenreceptor gene in metastatic androgen-independent prostate cancer. N Engl J Med. 1995;332(21):1393-1398.

9. Taplin ME, Bubley GJ, Ko YJ, et al. Selection for androgen receptor mutations in prostate cancers treated with androgen antagonist. Cancer Res. 1999;59(11):2511-2515.

10. Koivisto P, Kononen J, Palmberg C, et al. Androgen receptor gene amplification: a possible molecular mechanism for androgen deprivation therapy failure in prostate cancer. Cancer Res. 1997;57(2):314-319.
11. Gregory CW, He B, Johnson RT, et al. A mechanism for androgen receptor-mediated prostate cancer recurrence after androgen deprivation therapy. Cancer Res. 2001;61(11):4315-4319.

12. Montgomery RB, Mostaghel EA, Vessella R, et al. Maintenance of intratumoral androgens in metastatic prostate cancer: a mechanism for castration-resistant tumor growth. Cancer Res. 2008;68(11):4447-4454.

13. Cai C, Wang H, Xu Y, Chen S, Balk SP. Reactivation of androgen receptor-regulated TMPRSS2:ERG gene expression in castrationresistant prostate cancer. Cancer Res. 2009;69(15):6027-6032.

14. Katagiri M, Kagawa N, Waterman MR. The role of cytochrome b5 in the biosynthesis of androgens by human P450c17. Arch Biochem Biophys. 1995;317(2):343-347.

15. Dharia S, Slane A, Jian M, Conner M, Conley AJ, Parker CR Jr. Colocalization of P450c17 and cytochrome b5 in androgen-synthesizing tissues of the human. Biol Reprod. 2004;71(1):83-88.

16. Small EJ, Halabi S, Dawson NA, et al. Antiandrogen withdrawal alone or in combination with ketoconazole in androgen-independent prostate cancer patients: a phase III trial (CALGB 9583). J Clin Oncol. 2004;22(6):1025-1033.

17. Barrie SE, Potter GA, Goddard PM, Haynes BP, Dowsett M, Jarman M. Pharmacology of novel steroidal inhibitors of cytochrome P450(17) alpha (17 alpha-hydroxylase/C17-20 lyase). J Steroid Biochem Mol Biol. 1994;50(5-6):267-273.

18. O'Donnell A, Judson I, Dowsett M, et al. Hormonal impact of the 17alpha-hydroxylase/C(17,20)-lyase inhibitor abiraterone acetate (CB7630) in patients with prostate cancer. $\mathrm{Br} J$ Cancer. 2004;90(12):2317-2325.

19. Attard G, Reid AH, Yap TA, et al. Phase I clinical trial of a selective inhibitor of CYP17, abiraterone acetate, confirms that castrationresistant prostate cancer commonly remains hormone driven. J Clin Oncol. 2008;26(28):4563-4571.

20. Ryan CJ, Smith MR, Fong L, et al. Phase I clinical trial of the CYP17 inhibitor abiraterone acetate demonstrating clinical activity in patients with castration-resistant prostate cancer who received prior ketoconazole therapy. J Clin Oncol. 2010;28(9):1481-1488.

21. Attard G, Reid AH, A'Hern R, et al. Selective inhibition of CYP17 with abiraterone acetate is highly active in the treatment of castrationresistant prostate cancer. J Clin Oncol. 2009;27(23):3742-3748.

22. Reid AH, Attard G, Danila DC, et al. Significant and sustained antitumor activity in post-docetaxel, castration-resistant prostate cancer with the CYP17 inhibitor abiraterone acetate. J Clin Oncol. 2010;28(9):1489-1495.

23. Danila DC, Morris MJ, de Bono JS, et al. Phase II multicenter study of abiraterone acetate plus prednisone therapy in patients with docetaxel-treated castration-resistant prostate cancer. J Clin Oncol. 2010;28(9):1496-1501.

24. Ryan CJ, Shah S, Efstathiou E, et al. Phase II study of abiraterone acetate in chemotherapy-naive metastatic castration-resistant prostate cancer displaying bone flare discordant with serologic response. Clin Cancer Res. 2011;17(14):4854-4861.

25. Zhao XY, Malloy PJ, Krishnan AV, et al. Glucocorticoids can promote androgen-independent growth of prostate cancer cells through a mutated androgen receptor. Nat Med. 2000;6(6):703-706.

26. de Bono JS, Logothetis CJ, Molina A, et al. Abiraterone and increased survival in metastatic prostate cancer. $N$ Engl J Med. 2011;364(21):1995-2005.

\section{Publish your work in this journal}

Drug Design, Development and Therapy is an international, peerreviewed open-access journal that spans the spectrum of drug design and development through to clinical applications. Clinical outcomes, patient safety, and programs for the development and effective, safe, and sustained use of medicines are a feature of the journal, which

\section{Dovepress}

has also been accepted for indexing on PubMed Central. The manuscript management system is completely online and includes a very quick and fair peer-review system, which is all easy to use. Visit $\mathrm{http}: / /$ www.dovepress.com/testimonials.php to read real quotes from published authors. 